\title{
ON THE SUM OF TWO SOLID ALEXANDER HORNED SPHERES
}

\author{
BY \\ B. G. CASLER $\left({ }^{1}\right)$
}

1. Introduction. We use $E^{n}$ to denote Euclidean $n$-space, $S^{n}$ to denote an $n$-sphere in $E^{n+1}$, i.e., the set of all points in $E^{n+1}$ at a distance of 1 from the origin. $I^{n+1}$ is the closure of the bounded complementary domain of $S^{n}$ in $E^{n+1}$.

A set $U$ in $E^{n}$ is simply connected if each closed curve $J$ in $U$ can be shrunk to a point in $U$. That is, if $g$ is a continuous function from $\operatorname{Bd}\left(I^{2}\right)$ onto $J$, then $g$ may be extended to take all of $I^{2}$ into $U$. Each complementary domain of $S^{n}$ in $E^{n+1}$ is simply connected.

Alexander [1] described a simple surface, $M$ (a set homeomorphic to $S^{2}$ ), in $S^{3}$ such that one complementary domain, $U$, of $M$ in $S^{3}$ was not simply connected. Bing in [3] described a solid horned sphere as $M+U$; that is, Alexander's horned sphere, $M$, together with the nonsimply connected complementary domain, $U$, of $M$.

Bing proved in [3] that if two solid horned spheres were sewn together along the boundary of each with the identity homeomorphism, then the resulting continuum is homeomorphic to $S^{3}$.

Ball in [2] modified Alexander's example to obtain a horned sphere such that if two of Ball's solid horned spheres were sewn together in a particular way, the resulting continuum is not $S^{3}$. This was done as a partial answer to the question raised by Bing in [3], which may be roughly stated as follows: if two solid horned spheres are sewn together with an arbitrary homeomorphism, is the resultant continuum $S^{3}$ ? The theorem in $\$ 3$ states that this question has an affirmative answer if the horned sphere in question is the Alexander example.

We shall adopt the following notation. Suppose $b$ is a positive integer with $n$ digits, and each digit in $b$ is either a 1 or a 2 . Then we let $b=n \alpha$. (At times it will be necessary to distinguish two or more such positive integers. In this case we shall use $n \alpha, n \beta$, etc.) It follows that $1 \alpha$ is 1 or 2 and $2 \alpha$ is either $11,12,21$, or 22 . Suppose some $n \alpha$ is given and we wish to express the positive integer such that the first $n$ digits are exactly the

\footnotetext{
Presented to the Society, April 12, 1962; received by the editors December 30, 1963.

( ${ }^{1}$ ) This paper is the author's Doctoral Dissertation written at the University of Wisconsin under the supervision of Professor R. H. Bing whose interest, patience and numerous helpful suggestions were of invaluable assistance in its preparation. This research was supported under grant NSF G-11665 while the author was an assistant to R. H. Bing during the summers of 1960 and 1961 .
} 
same as $n \alpha$ and the last digit is a 1 . We call this integer $n \alpha 1$. If the last digit were a 2, then it would be represented by $n_{\alpha} 2$. For example let $n \alpha$ $=12112$, then $n \alpha 1=121121$ and $n \alpha 2=121122$. The positive integer $n$ contained in $n_{\alpha}$ is' important in the argument.

Further we shall assume that $\sum A_{n \alpha}$ means the union of all $A_{n}$ where $A_{n \alpha}$ denotes a set and $n \alpha$ runs through the set of all positive integers containing $n$ digits and each digit is a 1 or a 2 . In the same way $\left\{A_{n \alpha}\right\}$ is the collection of all $A_{n \alpha}$ where $n \alpha$ takes on all values.

While this notation is unfamiliar, it avoids double subscripts and some of the confusion resulting from notation.

Further if $A$ is a set, $\mathrm{Cl}(A)$ is the closure of $A, \operatorname{Int}(A)$ is the interior of $A$, and $\operatorname{Bd}(A)$ is the boundary of $A$. We let $d$ be the Euclidean metric for $E^{3}$. For $t$ real, let $a \leqq t \leqq b$ be $[a, b], a<t<b$ be $(a, b), a \leqq t<b$ be $[a, b)$, and $a<t \leqq b$ be $(a, b]$.

2. The horned sphere. The following definition of a solid horned sphere is due to Bing in [3]. The notation used in describing the horned sphere will be used in describing a homeomorphism of the sum of two solid horned spheres onto $S^{3}$.

Suppose $C$ is a right circular cylinder in $E^{3}$ with bases $D_{1}$ and $D_{2}$. Two mutually exclusive discs in $D_{1 \alpha}$ are replaced by the surfaces of tubes $T_{1 \alpha 1}$ and $T_{1 \alpha 2}$ and discs $D_{1 \alpha 1}$ and $D_{1 \alpha 2}$ as shown in Figure 1 where $D_{1 \alpha 1}$ and $D_{1 \alpha 2}$ are the bases of a right circular cylinder $C_{1 \alpha}$ and $D_{1}+T_{11}+C_{1}+T_{12}$ is hooked to $D_{2}+T_{21}+C_{2}+T_{22}$ as shown.

Discs in the bases of the cylinder $C_{1 \alpha}$ are replaced by the surfaces of tubes $T_{1 \alpha 11}, T_{1 \alpha 12}, T_{1 \alpha 21}$, and $T_{1 \alpha 22}$ and discs $D_{1 \alpha 11}, D_{1 \alpha 12}$, and $D_{1 \alpha 22}$ as before. The process is continued to get the horned sphere $M$. We use $M_{1}$ to denote the part of $M$ which is the closure of the part of $M$ on the exterior of $C_{1}+C_{2}, M_{1}=C-\sum$ (discs cut from $\left.D_{1}+D_{2}\right)+\sum T_{2 \alpha}$. Likewise, $M_{n}$ denotes the closure of the part of $M$ on the exterior of $\sum C_{n \alpha}$. It is topologically equivalent to $S^{2}$ minus $2^{n+1}$ open discs. Let $M_{0}$ be the Cantor set $M-\sum_{n=1}^{\infty} M_{n}$.

Although $M$ is homeomorphic to $S^{2}$, its interior $U$ is not simply connected [4]. We call this complementary domain of $M$ which is not simply connected the bad complementary domain of $M$. A horned sphere plus its bad complementary domain is called a solid horned sphere. The part of $U$ which is on the exterior of $\sum C_{n \alpha}$ is denoted by $U_{n}$.

3. The sum of two solid horned spheres. We now state our main result in the following theorem, which shows the effect of sewing two solid horned spheres together.

Theorem. A continuum is homeomorphic with $S^{3}$ if it is the sum of three mutually exclusive sets $M, U^{1}$, and $U^{2}$ such that there is a homeomorphism 


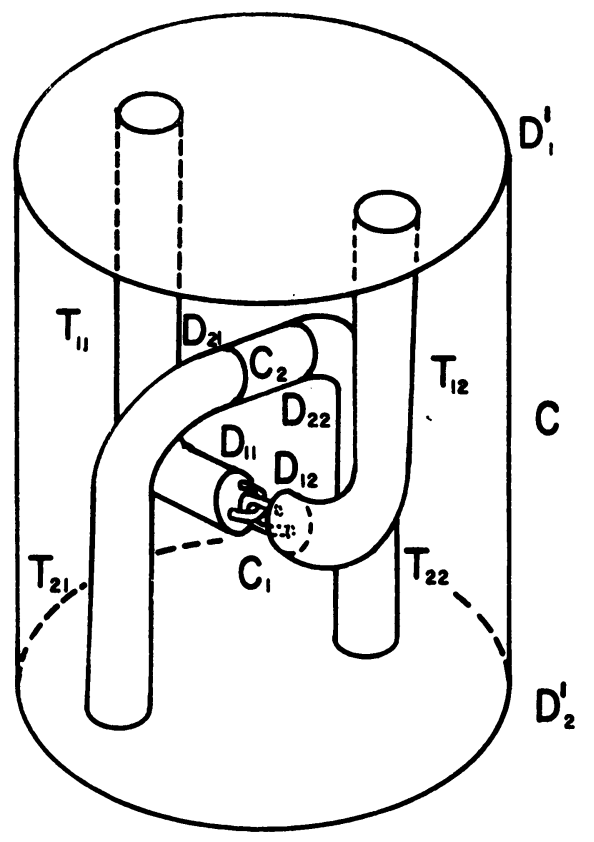

Figure 1

of $M+U^{i}, i=1,2$, onto a solid horned sphere that carries $M$ onto a horned sphere.

We defer the proof of the Theorem until $\S 5$.

4. A decomposition of $E^{3}$. Suppose $g$ is any homeomorphism of $M$ onto $M$. There is a continuum $\left(M+U^{1}+U^{2}\right)_{g}$ and homeomorphisms $g_{1}$ and $g_{2}$ such that $g_{i}$ takes $M+U^{i}, i=1,2$, onto a solid horned sphere and such that $\left(g_{2} \mid M\right)\left(g_{1} \mid M\right)^{-1}=g$. Now suppose $h$ is a homeomorphism from $M$ onto $M$ and $\left(M+U^{1}+U^{2}\right)_{h}$ is defined as above. Further suppose that there is a topological 2-cell $W$ containing $M_{0}+g\left(M_{0}\right)$ contained in $M$ such that $g|W=h| W$. It is easy to see that $\left(M+U^{1}+U^{2}\right)_{g}$ is homeomorphic to $\left(M+U^{1}+U^{2}\right)_{h}$.

As $M_{0}+g\left(M_{0}\right)$ is a Cantor set in $M$, a topological 2-sphere, there is a point $y$ contained in $M-\left(M_{0}+g\left(M_{0}\right)\right)$ such that $g(y)$ is contained in $M-\left(M_{0}+g\left(M_{0}\right)\right)$. There is a topological 2-cell $W$ contained in $M$ containing $M_{0}+g\left(M_{0}\right)$ such that the points $y$ and $g(y)$ are contained in $M-W$. As $\mathrm{Cl}(M-W)$ is a topological 2-cell, there is a homeomorphism $h$ of $M$ onto $M$ such that $h(y)=y$ and $g|W=h| W$. It follows that we may assume without loss of generality that $g$ has a fixed point, $y$, in $M-M_{0}$.

We now slightly modify the procedure of Bing in [3]. Suppose $P$ is the 
$x y$ plane in $E^{3}$, i.e., $P=\left\{x, y, z \in E^{3} \mid z=0\right\}$. Suppose $\operatorname{Cl}\left(A_{1}\right)$ and $\operatorname{Cl}\left(A_{2}\right)$ are two solid tori contained in $E^{3}$ as used by Bing, such that each is symmetric with respect to $P$ and the boundary, $\operatorname{Cl}\left(A_{i}\right)-A_{i}$, of $A_{i}$ is a 2dimensional torus which intersects $P$ in two circles. We use $L$ to denote that part of $E^{3}$ where $z$ is positive, i.e., $\left\{x, y, z \in E^{3} \mid z>0\right\}$, and $R$ to denote that part of $E^{3}$ where $z$ is negative, i.e., $\left\{x, y, z \in E^{3} \mid z<0\right\}$.

We shall now describe a decomposition of $E^{3}$. As we describe that part of the decomposition that intersects $\mathrm{Cl}(L)$ we shall describe a homeomorphism from $M+U-\left(M_{0}+y\right)$ into $\mathrm{Cl}(L)$. The notation is the same as used in \$2.

There is a homeomorphism $F_{1}$ of $\mathrm{Cl}\left(U_{1}\right)$ onto $\mathrm{Cl}(L)-\left(A_{1}+A_{2}\right)$ such that $F_{1}\left(M_{1}-y\right)=P-\left(A_{1}+A_{2}\right)$ and $F_{1}\left(\mathrm{Cl}(U) \cdot C_{1 \alpha}\right)=\mathrm{Cl}(L) \cdot\left(\mathrm{Cl}\left(A_{1 \alpha}\right)\right.$ $\left.-A_{1 \alpha}\right)$. Let $L A_{1 \alpha}=\mathrm{Cl}\left(A_{1 \alpha}\right) \cdot \mathrm{Cl}(L)$, as shown in Figure 2.

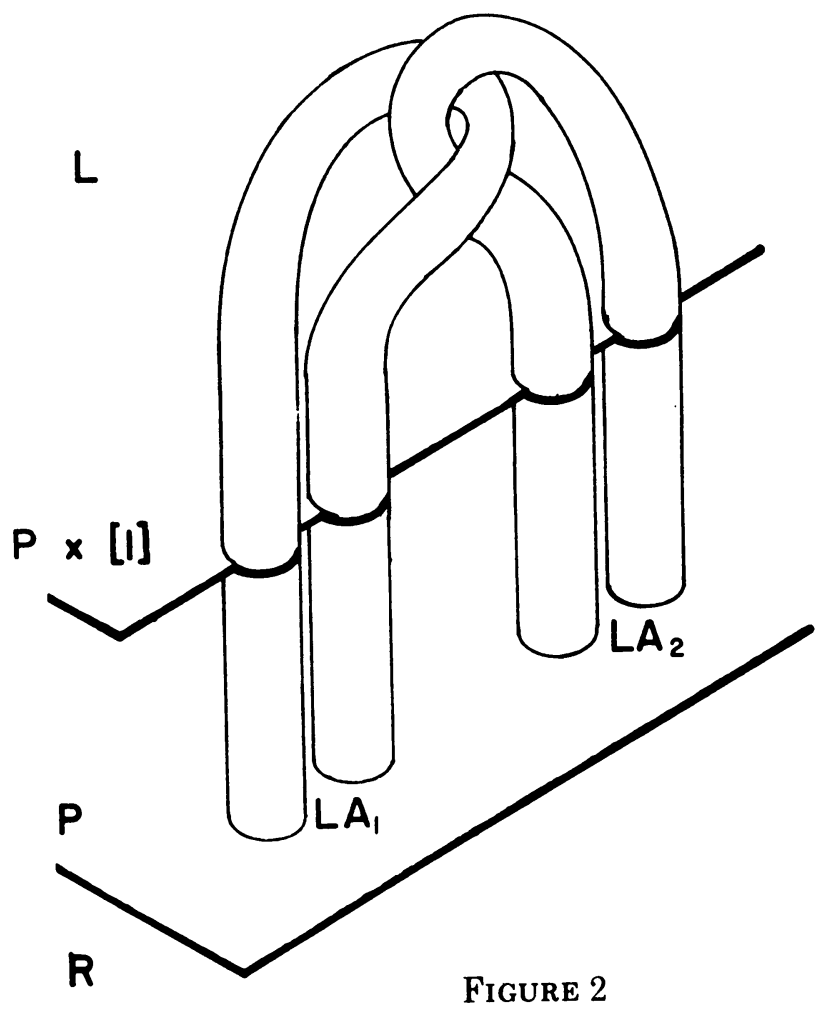

Consider $A_{1 \alpha}$. Following Bing's procedure [3], put a chain of two solid tori, $\mathrm{Cl}\left(A_{1 \alpha 1}\right)+\mathrm{Cl}\left(A_{1 \alpha 2}\right)$, in $A_{1 \alpha}$ so that each is symmetric with $P$. There is a homeomorphism $F_{2}$ of $\mathrm{Cl}\left(U_{2}\right)$ onto $\mathrm{Cl}(L)-\sum A_{2 \alpha}$ that preserves $F_{1}$ on $\mathrm{Cl}\left(U_{2}\right)$ such that $F_{2}\left(\mathrm{Cl}\left(U_{2}\right) \cdot C_{2 \alpha}\right)=\mathrm{Cl}(L) \cdot\left(\mathrm{Cl}\left(A_{2 \alpha}\right)-A_{2 \alpha}\right)$. Let $L A_{2 a}$ $=\mathrm{Cl}(L) \cdot \mathrm{Cl}\left(A_{2 \alpha}\right)$, as shown in Figure 3 . 


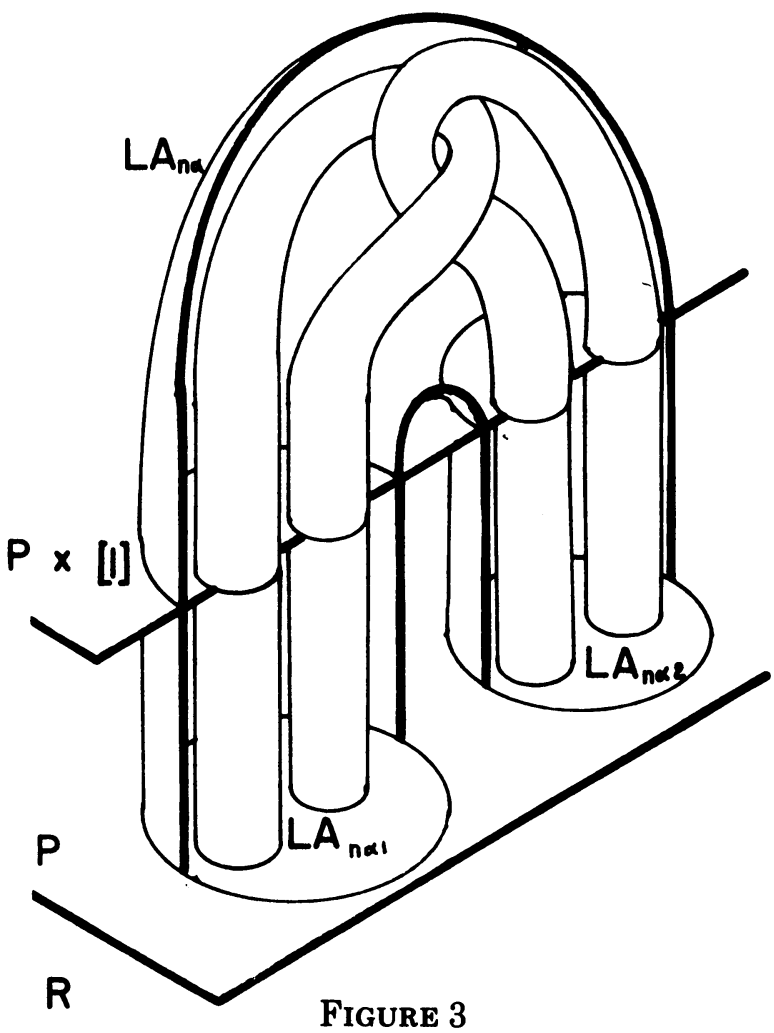

The process is continued. We get more solid tori, more $F_{i}$ 's, and more $L A_{i \alpha}$ 's. We use $L A_{0}$ to denote $\prod_{i=1}^{\infty} \sum L A_{i \alpha}$.

The tori could have been defined so that each component of $L A_{0}$ is an arc which intersects no plane parallel to $P$ in two points and so that part of $L A_{i \alpha}$ between $P$ and a plane parallel to $P$ one unit from $P$ in $L$ is the union of two perpendicular solid cylinders, as shown in Figures 2 and 3. We therefore assume this was done and that each component of $L A_{0}$ is an arc intersecting $P$ in an end point.

The sequence $F_{1}, F_{2}, \ldots$ describes a homeomorphism $F$ between $M+U$ $-\left(M_{0}+y\right)$ and $\mathrm{Cl}(L)-L A_{0}$. There is a homeomorphism $f$ of $M-y$ onto $P$ such that $f\left|M-M_{0}=F\right| M-M_{0}$.

Let $\phi$ be a homeomorphism of $\mathrm{Cl}(L)$ onto $\mathrm{Cl}(R)$ such that $\phi(x, y, z)$ $=\left(f g f^{-1}(x, y),-z\right)$, for $z \geqq 0$, where $(x, y)$ denotes the point of $E^{3},(x, y, 0)$. The homeomorphism $\phi$ is well defined as $f g f^{-1}$ is a homeomorphism of $P$ onto itself. The homeomorphism $\phi$ maps any ray in $\mathrm{Cl}(L)$ perpendicular to $P$ and with end point in $P$ onto a ray in $\mathrm{Cl}(R)$ perpendicular to $P$ and with end point in $P$. Let $R A_{i \alpha}=\phi\left(L A_{i \alpha}\right)$, and $R A_{0}=\prod_{i=1}^{\infty} \sum R_{i \alpha}=\phi\left(L A_{0}\right)$. Let $G=\phi F$. The homeomorphism $G$ describes a homeomorphism from $M+U-\left(M_{0}+y\right)$ into $\mathrm{Cl}(R)$. 
Now consider $\left(M+U^{1}+U^{2}\right)_{g}$ and the functions $g_{1}$ and $g_{2}$ defined in the first paragraph of this section. We now define a homeomorphism $S$ as follows. Let $F g_{1}\left|U^{1}=S\right| U^{1}$ and $G g_{2}\left|U^{2}=S\right| U^{2}$. As $F, G, g_{1}$, and $g_{2}$ are homeomorphisms and $U^{1}$ and $U^{2}$ are disjoint open sets, $S$ is a homeomorphism of $U^{1}+U^{2}$ onto $L+R-\left(L A_{0}+R A_{0}\right)$. Let $\left\{u_{i}\right\}$ be any sequence of points in $U^{1}+U^{2}$ converging to a point $u \neq y$ contained in $\left(M+U^{1}+U^{2}\right)_{g}$. Let $S(u)=\operatorname{Lim} S\left(u_{i}\right)$. It follows that $S$ is a homeomorphism from $\left(M+U^{1}+U^{2}\right)_{g}-y$ onto the decomposition space $X$ whose points are the points of $E^{3}-\left(L A_{0}+R A_{0}\right)$ and the components of $L A_{0}+R A_{0}$.

5. The shrinking of the components of $L A_{0}+R A_{0}$. If $S$ could have been been defined so that $S\left(M_{0}+g\left(M_{0}\right)\right)$ is a Cantor set of points rather than a Cantor set of arcs, then $S$ would be a homeomorphism from $\left(M+U^{1}+U^{2}\right)_{g}$ $-y$ onto $E^{3}$ and with the one point compactification of $E^{3}$ the proof of the Theorem would be complete. One way to prove the Theorem is to define a continuous function, $T$, from $E^{3}$ onto $E^{3}$ such that $T$ shrinks each distinct component of $L A_{0}+R A_{0}$ onto a distinct point of $E^{3}$ and is a homeomorphism of $E^{3}-\left(L A_{0}+R A_{0}\right)$. It follows that $S$ composed with $T$ is a homeomorphism of $\left(M+U^{1}+U^{2}\right)_{g}-y$ onto $E^{3}$. We shall not define the function $T$ directly but define a sequence of homeomorphisms, $T_{i}$, that will eventually shrink the components of $L A_{0}+R A_{0}$.

The following lemma will allow us to define the $T_{i}$ 's. However before stating the lemma let us make the following definition. Suppose $\epsilon$ is a positive number. Let $\sum L A_{n \alpha}(\epsilon)=\left(\sum L A_{n \alpha}\right) \cdot P \times[-\epsilon, 0]$ where $[-\epsilon, 0]$ is an interval on the $z$ axis of $E^{3}$. In the same way let $\sum R A_{n \alpha}(\epsilon)=\left(\sum R A_{n \alpha}\right)$ . $P \times[0, \epsilon]$. The set $\sum L A_{n \alpha}(\epsilon)$ is a small extension of $\sum L A_{n \alpha}$ into $R$.

LEMma 1. For each $\epsilon>0$ and each pair of positive integers $p$ and $q$ there is a pair of positive integers $r$ and $s$ and a homeomorphism $T^{\prime}$ of $E^{3}$ onto $E^{3}$ fixed on $E^{3}-\left(\sum L A_{p \alpha}+\sum R A_{q \alpha}+\sum L A_{p \alpha}(\epsilon)+\sum R A_{q \alpha}(\epsilon)\right)$ and taking each component of $\sum L A_{r \alpha}+\sum R A_{s \alpha}$ into a set of diameter less than $\epsilon$.

Proof. The proof of this lemma will be simplified if we make the following assumptions. We will show that these assumptions are valid in $\$ 6$.

A(1) For each pair of positive integers $p$ and $q$ there is a pair of positive integers $p^{*}$ and $q^{*}, p \leqq p^{*}, q \leqq q^{*}$, such that:

(i) each component of $\left(\sum L A_{p^{*} \alpha}+\sum R A_{q^{*} \alpha}\right) \cdot P$ is of diameter $\epsilon / 5$.

(ii) each component of $\sum L A_{\left(p^{*}+1\right) \alpha}+\sum R A_{\left(q^{*}+1\right) \alpha}$ contains at most one component of $\sum L A_{\left(p^{*}+1\right) \alpha}$.

A(2) For each pair of positive integers $p$ and $q$ there is a pair of positive integers $p^{\prime}$ and $q^{\prime}, p^{\prime}>p+1, q^{\prime}>q+1$, and a finite set of disks, $B_{1}$, $B_{2}, \cdots, B_{k}$ contained in $P$ such that,

(i) $\left(\sum L A_{p^{\prime} \alpha}+\sum R A_{q^{\prime} \alpha}\right) \cdot P$ is contained in $\operatorname{Int}\left(\sum_{i=1}^{k} B_{i}\right)$,

(ii) at most one component of $\sum L A_{p^{\prime} \alpha}$ intersects $B_{i}, i=1,2, \cdots, k$, 
(iii) no component of $\sum L A_{p^{\prime} \alpha}$ or $\sum R A_{q^{\prime} \alpha}$ intersects more than one of the disks $B_{1}, B_{2}, \ldots, B_{k}$,

(iv) if $B_{i}$ intersects both $\sum L A_{p^{\prime} \alpha}$ and $\sum R A_{q^{\prime} \alpha}$, then $B_{i}$ is contained in $\sum \operatorname{Int}\left(\left(R A_{(q+2)_{\alpha}}\right) \cdot P\right)$,

(v) if $B_{i}$ intersects both $\sum L A_{p^{\prime} \alpha}$ and $\sum R A_{q^{\prime} \alpha}$, then $B_{i}$ is contained in $\sum \operatorname{Int}\left(\left(L A_{(p+1) \alpha}\right) \cdot P\right)$.

A(3) $\left({ }^{2}\right)$ For each $\epsilon>0$ and each pair of positive integers $p$ and $q$ there is a homeomorphism, $H_{0}$, of $E^{3}$ onto $E^{3}$ and a finite sequence of planes, $P_{1}, P_{2}, \ldots, P_{m}$, such that:

(i) the distance from $P$ to $P_{i}$ is less than $1, i=1,2, \cdots, m$,

(ii) $P_{i}$ is parallel to $P, i=1,2, \cdots, m$,

(iii) $P_{1}$ and $P_{2}$ are in $L$,

(iv) $P_{(m-1)}$ and $P_{m}$ are in $R$,

(v) $P_{i}$ is between $P_{(i-1)}$ and $P_{(i+1)}, i=2,3, \cdots,(m-1)$,

(vi) $H_{0}$ is fixed on $E^{3}-\left(\sum L A_{p \alpha}+\sum R A_{q \alpha}\right)$,

(vii) the diameter of each component of

$$
H_{0}\left(\sum L A_{(p+1) \alpha}+\sum R A_{(q+1) \alpha}-\sum_{i=1}^{m} P_{i}\right)
$$

is less than $\epsilon / 5$.

A(4) Suppose $p$ and $p^{\prime}$ are the positive integers described in $A(2), P_{1}$ and $P_{2}$ are the planes described in $A(3)$, and $L A_{p^{\prime} \alpha}$ is contained in $L A_{(p+1) \alpha}$. There is a homeomorphism $H_{1}$, of $E^{3}$ onto $E^{3}$ such that:

(i) $H_{1}$ is fixed on that part of $L A_{(p+1) \alpha}$ that is between $P_{2}$ and $P$,

(ii) $H_{1}$ is fixed on that part of $L A_{p^{\prime} \alpha}$ that is between $P_{1}$ and $P$,

(iii) $H_{1}$ is fixed on $E^{3}-L A_{(p+1) \alpha}$,

(iv) $H_{1}$ takes each $L A_{p^{\prime} \beta} \alpha \neq \beta$, contained in $L A_{(p+1) \alpha}$ into a set that does not intersect $P_{1}$.

A(5) Suppose $q$ is the positive integer of $\mathrm{A}(2), P_{m}$ and $P_{(m-1)}$ are the planes of $\mathrm{A}(3)$, and $R A_{(q+1) \alpha}$ is given. Then there is a homeomorphism $\mathrm{H}_{2}$ of $E^{3}$ onto $E^{3}$ such that:

(i) $\mathrm{H}_{2}$ is fixed on that part of $R A_{(q+1) \alpha}$ between $P_{(m-1)}$ and $P$,

(ii) $H_{2}$ is fixed on that part of $R A_{(q+1) \alpha 1}$ between $P_{m}$ and $P$,

(iii) $H_{2}$ is fixed on $E^{3}-R A_{(q+1) \alpha}$,

(iv) $H_{2}\left(R A_{(q+1) \alpha 2}\right)$ does not intersect $P_{m}$.

Suppose that there is a homeomorphism $T^{\prime}$ of $E^{3}$ onto $E^{3}$ fixed on $E^{3}$ $-\left(\sum L A_{p^{*} \alpha}+\sum R A_{q^{*} \alpha}+\sum L A_{p^{*} \alpha}(\epsilon)+\sum R A_{q^{*} \alpha}(\epsilon)\right)$. If $p^{*} \geqq p$ and $q^{*} \geqq q$, then $T^{\prime}$ is fixed on $E^{3}-\left(\sum L A_{p \alpha}+\sum R A_{q \alpha}+\sum L A_{p \alpha}(\epsilon)+\sum R A_{q \alpha}(\epsilon)\right)$.

$\left({ }^{2}\right)$ We note that the procedure at this point differs from Bing's construction in [3] in the following way. Bing used the planes $P_{1} \ldots P_{m}$ to partition his sets into "small" pieces whereas the author used $H_{0}\left(\sum_{i=1}^{m} P_{i}\right)$ to partition his sets. It will be noted that there are some large components of $\left(\sum L A_{p_{\alpha}}+\sum R A_{q_{\alpha}}\right)-\sum_{i=1}^{m} P_{i}$ but there are no large components of $H_{0}\left(\left(\sum L A_{(p+1) \alpha}+\sum R A_{(q+1) \alpha}\right)-\sum_{i=1}^{m} P_{i}\right)$. 
It follows that we may assume without loss of generality that $p=p^{*}$ and $q=q^{*}$ where $p^{*}$ and $q^{*}$ are the positive integers promised by $\mathrm{A}(1)$.

Now suppose that there is a homeomorphism $H$ of $E^{3}$ onto $E^{3}$ fixed on $E^{3}-\left(\sum L A_{(p+1) \alpha}+\sum R A_{(q+1) \alpha}+\sum L A_{(p+1) \alpha}(\epsilon)+\sum R A_{(q+1) \alpha}(\epsilon)\right)$ taking each component of $\sum L A_{r \alpha}+\sum R A_{s \alpha}$ into a set that intersects at most five components of $\left(\sum L A_{(p+1) \alpha}+\sum R A_{(q+1) \alpha}\right)-\sum_{i=1}^{m} P_{i}$ where $P_{1}, \cdots, P_{m}$ are the planes of $\mathrm{A}(3)$. By the properties of $H_{0}$ given in $\mathrm{A}(3)$, the homeomorphism $H_{0} H$ is the required homeomorphism $T^{\prime}$.

We shall now construct the homeomorphism $H$ inductively using the number of planes in A(3). Let $m=4, P_{1}$ and $P_{2}$ be in $L$ and $P_{3}$ and $P_{4}$ be in $R$. Let $p^{\prime}, q^{\prime}$ and $B_{1}, \cdots, B_{k}$ be the positive integers and disks promised by $A(2)$. Let $B_{1}, B_{2}, \cdots, B_{n}$ be the disks in $B_{1}, \cdots, B_{k}$ that intersect $\sum L A_{p^{\prime} \alpha}$ and $\sum R A_{q^{\prime} \alpha}$. $\mathrm{By} \mathrm{A}(2)$ (iv) and $\mathrm{A}(2)$ (v), $\sum_{i=1}^{n} B_{i}$ is a subset of $\sum L A_{(p+1) \alpha}$ $+\sum R A_{(q+1) \alpha}$. Let $K_{1}, K_{2}, \cdots, K_{u}$ be the components of $\sum L A_{(p+1) \alpha}$ $+\sum R A_{(q+1) \alpha}$ and $Q_{1,1}, Q_{1,2}, \cdots, Q_{2,1}, \cdots, Q_{u, v}$ be the components of $\sum L A_{p^{\prime} \alpha}$ $+\sum R A_{q^{\prime} \alpha}+\sum_{i=1}^{n} B_{i}$ where $Q_{i, j}$ is a subset of $K_{i}$.

Note that the set $\left(\sum L A_{p^{\prime} \alpha}+\sum R A_{q^{\prime} \alpha}\right) \cdot P$ may be a very complicated set. We use the disks $B_{1}, B_{2}, \cdots, B_{n}$ to expand $\left(\sum L A_{p^{\prime} \alpha}+\sum R A_{q^{\prime} \alpha}\right) \cdot P$ into a more manageable set.

If a component $L A_{p^{\prime} \alpha}$ of $\sum L A_{p^{\prime} \alpha}$ does not intersect a $B_{i}, i \leqq n$, then $L A_{p^{\prime} \alpha}$ does not intersect $\sum R A_{q^{\prime} \alpha}$. There is a natural method to shrink $L A_{p^{\prime} \alpha}$ leaving $E^{3}-\left(\sum L A_{p \alpha}+\sum L A_{p \alpha}(\epsilon)\right)$ fixed. In the same way if $R A_{q^{\prime} \alpha}$ does not intersect a $B_{i}, i \leqq n$, then $R A_{q^{\prime}}$ may be shrunk. With this note we ignore sets of this type as they give no difficulty in the shrinking process.

So we may assume $Q_{1,1}$ contains a component of $\sum L A_{p^{\prime} \alpha}$, say $L A_{p^{\prime} \alpha}$. There is a homeomorphism $H_{1}$ as defined in $\mathrm{A}(4)$. By $\mathrm{A}(1)$ (ii), $K_{1}$ contains exactly one component of $\sum L A_{(p+1) \alpha}$. Hence $H_{1}\left(Q_{1,1}\right)$ intersects $P_{1}$ and $H_{1}\left(Q_{1, j}\right), j \neq 1$, does not intersect $P_{1}$.

By $\mathrm{A}(2)$ (iii), $Q_{1,1}$ contains exactly one disk $B_{i}, i \leqq n$. By $\mathrm{A}(2)$ (iv), $B_{i}$ is contained in $\operatorname{Int}\left(\left(R A_{(q+2) \alpha}\right) \cdot P\right)$, where $R A_{(q+2) \alpha}$ is a component of $\sum R A_{(q+2) \alpha}$. Let $R A_{(q+2) \alpha}=R A_{(q+1) \alpha 2}$. By construction $\left(Q_{1,1}\right) \cdot R$ is a subset of $R A_{(q+1) \alpha 2}$. There is a homeomorphism $H_{2}$ as defined in $\mathrm{A}(5)$. Note $H_{1}$ is fixed on $R$ and $H_{2}$ is fixed on $L$. Further $H_{2}\left(R_{(q+1) \alpha 2}\right)$ does not intersect $P_{4}$ and $\left(Q_{1,1}\right) \cdot P$ is contained in $R A_{(q+1) \alpha 2}$. It follows that for each integer $j, H_{2} H_{1}\left(Q_{1, j}\right)$ intersects at most three of the four planes $P_{1}, P_{2}, P_{3}$, and $P_{4}$. Further by $\mathrm{A}(4)$ and $\mathrm{A}(5), H_{2} H_{1}$ is a homeomorphism of $E^{3}$ onto $E^{3}$ fixed on $E^{3}-\left(\sum L A_{(p+1) \alpha}+\sum R A_{(q+1) \alpha}\right)$.

Without loss of generality we may assume that $H_{1}$ and $H_{2}$ have been defined for each $K_{i}, i=1,2, \cdots, u$, and that $H_{2} H_{1}\left(Q_{i, j}\right)$ intersects at most three of the four planes $P_{1}, P_{2}, P_{3}, P_{4}$ for each $i, j$. In this case let $H=H_{2} H_{1}$.

To complete the proof of this case we must show that no component of $H\left(\sum L A_{p^{\prime} \alpha}+\sum R A_{q^{\prime} \alpha}\right)$ intersects more than five components of 
$\left(\sum L A_{(p+1) \alpha}+\sum R A_{(q+1) \alpha}\right)-\sum_{i=1}^{4} P_{i} . \quad \mathrm{By} \quad \mathrm{A}(2) \quad$ (iv) we know that $\sum_{i=1}^{n} B_{i}$ is contained in $\sum \operatorname{Int}\left(\left(R A_{(q+2) \alpha}\right) \cdot P\right)$ and hence each component $Q_{i, j}$ intersects at most one component of $\left(\sum R A_{(p+1)_{a}}\right) \cdot P$. By $\mathrm{A}(2)$ (v) $\sum_{i=1}^{n} B_{i}$ is contained in $\operatorname{Int}\left(\left(L A_{(p+1)_{\alpha}}\right) \cdot P\right)$. Hence each component $Q_{i, j}$ intersects at most one component of $\left(\sum L A_{(p+1) \alpha}\right) \cdot P$.

Conditions $A(2)$ (iv) and $A(2)$ (v) together imply that $B_{i} \times[-1,1]$, $i \leqq n$, is contained in $\operatorname{Int}\left(L A_{(p+1) \alpha}+R A_{(q+1) \alpha}\right)$. By $\mathrm{A}(4)$ and $\mathrm{A}(5), H$ is fixed between the planes $P_{2}$ and $P_{3}$. As $H$ is fixed between the planes $P_{2}$ and $P_{3}$, no component of $H\left(\sum L A_{p^{\prime} \alpha}+\sum R A_{q^{\prime} \alpha}\right)$ can intersect more than one component of $\sum L A_{(p+1) \alpha}-\left(P_{1}+P_{2}\right)$ that intersects $P$ or more than one component of $\sum R A_{(q+1) \alpha}-\left(P_{3}+P_{4}\right)$ that intersects $P$. Further for each $H\left(Q_{i, j}\right)$ there is a pair of components $L A_{(p+1) \alpha}$ and $R A_{(q+1) \alpha}$ such that $H\left(Q_{i, j}\right)$ is contained in $L A_{(p+1) \alpha}+R A_{(q+1) \alpha}$. As $H\left(Q_{i, j}\right)$ can intersect either at most $P_{1}, P_{2}$, and $P_{3}$ or at most $P_{2}, P_{3}$, and $P_{4}$ it follows that $H\left(Q_{i, j}\right)$ can intersect at most five components of $\left(L A_{(p+1) \alpha}+R A_{(q+1) \alpha}\right)-\sum_{i=1}^{4} P_{i}$ as is shown in Figure 4, and the lemma follows from this case.

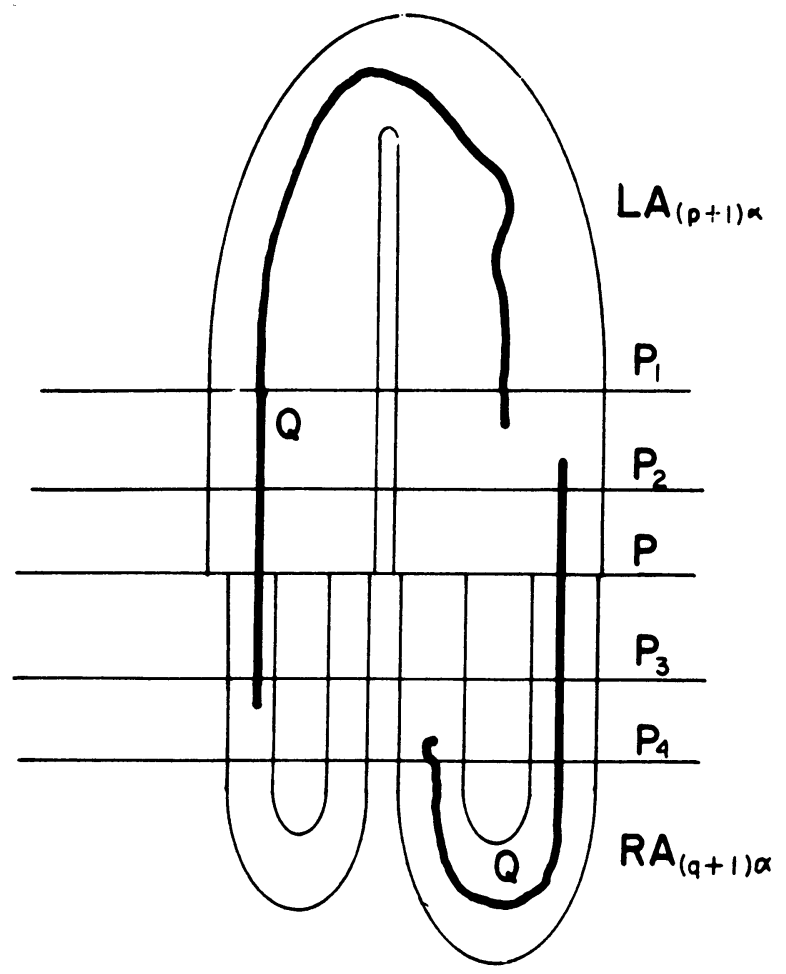

Figure 4

We proceed to the case where the number of planes is five, $P_{1}$ and $P_{2}$ are contained in $L, P_{4}$ and $P_{5}$ are contained in $R$ and $P_{3} \neq P$. Let us proceed 
as above where there are four planes by first choosing integers $p^{\prime}$ and $q^{\prime}$, constructing the $B_{i}$ 's and defining a homeomorphism $H_{1}$ and a homeomorphism $H_{2}$ using $P_{4}$ in place of $P_{3}$ and $P_{5}$ in place of $P_{4}$.

By $A(2)$ for the pair of positive integers $p^{\prime}$ and $q^{\prime}$ there is a pair of positive integers $p^{\prime \prime}$ and $q^{\prime \prime}$ and a finite set of disks $B_{1}^{\prime}, B_{2}^{\prime} \ldots B_{k^{\prime}}^{\prime}$ with the properties given in $\mathrm{A}(2)$ with appropriate changes in notation. Let $Q$ be any component of $H_{2} H_{1}\left(\sum L A_{p^{\prime} \alpha}+\sum R A_{q^{\prime} \alpha}\right)$ that intersects four of the five planes $P_{1}, \cdots, P_{5}$. As $Q$ is connected either $Q$ intersects $P_{1}, P_{2}, P_{3}$, and $P_{4}$ or $Q$ intersects $P_{2}, P_{3}, P_{4}$, and $P_{5}$.

Suppose $Q$ intersects $P_{1}, P_{2}, P_{3}$, and $P_{4}$ and $P_{3}$ is between $P$ and $P_{4}$. By $\mathrm{A}(4)$ (i) and $\mathrm{A}(4)$ (ii) there are exactly two components $R_{1}$ and $R_{2}$ of $Q-\left(P_{1}+P\right)$ whose closures intersect both $P_{1}$ and $P$. By construction $\mathrm{Cl}\left(R_{i}\right), i=1,2$, is a right circular cylinder. In $\$ 6$ we shall show that under this condition we may define a homeomorphism $H_{1}^{\prime}$ similar to $H_{1}$ and with the properties of $H_{1}$ given in $\mathrm{A}(4)$ with appropriate changes in notation.

Further by $A(5)$ (i) each component of $Q-\left(P_{4}+P\right)$ whose closure intersects both $P$ and $P_{4}$ is a right cylinder. As $P_{3}$ is between $P$ and $P_{4}$, $P_{3}$ cuts each component of $Q-\left(P_{4}-P\right)$ in a disk. In $\$ 6$ we shall show that under these conditions we may define a homeomorphism, $H_{2}^{\prime}$, similar to $H_{2}$ and with the properties of $H_{2}$ given in $\mathrm{A}(5)$ with appropriate changes in notation. It follows that each component of

$$
H_{2}^{\prime} H_{1}^{\prime}\left(\left(H_{1} H_{2}\left(\sum L A_{p^{\prime \prime} \alpha}+\sum R A_{q^{\prime \prime} \alpha}\right)\right) \cdot Q\right)
$$

intersects at most three of the five planes $P_{1}, \cdots, P_{5}$.

Now suppose $Q$ intersects $P_{2}, P_{3}, P_{4}$, and $P_{5}$ and $P_{3}$ is between $P_{2}$ and $P$. We note that the closure of the two components of $Q-\left(P_{2}+P\right)$ whose closures intersect both $P_{2}$ and $P$ are right circular cylinders, and $P_{3}$ cuts each of these components in a disk. Further we note that the closure of each component of $Q-\left(P+P_{3}\right)$ whose closures intersect both $P$ and $P_{5}$ is a right cylinder. It follows by the remarks above that we may define a homeomorphism, $H_{1}^{\prime}$ and a homeomorphism $H_{2}^{\prime}$ such that

$$
H_{2}^{\prime} H_{1}^{\prime}\left(\left(H_{2} H_{1}\left(\sum L A_{p^{\prime \prime} \alpha}+\sum R A_{q^{\prime \prime} \alpha}\right)\right) \cdot Q\right)
$$

intersects at most three of the five planes $P_{1}, \ldots, P_{5}$.

Now $P_{3}$ can not be both between $P$ and $P_{2}$ and between $P$ and $P_{4}$. But actually all of $P$ which we use here is $P \cdot Q$. The obvious procedure is to move $P \cdot Q$ between the "right" two planes and keep the geometry which will allow us to define $H_{1}^{\prime}$ and $H_{2}^{\prime}$.

Suppose $Q \cdot\left(H_{2} H_{1}\left(L A_{p^{\prime} \alpha}\right)\right) \neq \emptyset$ and $Q \cdot\left(H_{2} H_{1}\left(R A_{q^{\prime} \alpha}\right)\right) \neq \emptyset$. (The procedure in the contrary case is obvious.) Then $P \cdot Q$ is contained in $B_{1}^{\prime}, i \leqq n$. By construction $B_{i}^{\prime} \times\left[t_{1}, t_{5}\right]$ is contained in $\operatorname{Int}\left(\sum L A_{(p+1) \alpha}+\sum R A_{(q+1) \alpha}\right)$ where $t_{1}$ is the distance from $P$ to $P_{1}$ and $-t_{5}$ is the distance from $P$ to $P_{5}$. 
As $P \cdot Q$ is contained in Int $B_{i}^{\prime}$ there is a polyhedral disk $Y_{i}$ contained in $\left.Y_{i}+B_{i}^{\prime}\right)$ such that $\left(B_{i}^{\prime}-Y_{i}\right) \cdot\left(\sum L A_{p^{\prime} \alpha}+\sum R A_{q^{\prime} \alpha}\right)=\emptyset$. Let $\gamma$ be the least integer such that $Q$ intersects $P_{\gamma}$ and $\eta$ be the greatest integer such that $Q$ intersects $P_{\eta}$. (In this case $\gamma$ is either 1 or 2 and $\eta$ is either 4 or 5.) Let $t_{j}$ be a real number such that $P \times\left[t_{j}\right]$ is a plane between $P_{\gamma+1}$ and $P_{\eta-1}$. There is a piece-wise linear homeomorphism, $H_{3}$, from $E^{3}$ onto $E^{3}$ such that:

(i) $H_{3}$ is fixed on $E^{3}-\left(B_{i}^{\prime} \times\left[t_{\gamma}, t_{\eta}\right]\right)$ where $t_{\gamma}$ is the distance of $P_{\gamma}$ from $P$ and $-t_{\eta}$ is the distance from $P_{\eta}$ to $P$,

(ii) if $H_{3}(x) \neq x$, then the line through $H_{3}(x)$ and $x$ is perpendicular to $P$,

(iii) $H_{3}\left(Y_{i} \times[t]\right), t$ a real number, is contained in a plane parallel to $P$,

(iv) $H_{3}\left(Y_{i}\right)=Y_{i} \times\left[t_{j}\right]$.

Suppose $H_{2} H_{1}\left(L A_{p^{\prime} \alpha}\right)$ is contained in $Q$. Then $\left(H_{3} H_{2} H_{1}\left(L A_{p^{\prime} \alpha}\right)\right)$ $\left(B_{i} \times\left[t_{\gamma}, t_{\eta}\right]\right)=H_{3}\left(\left(L A_{p^{\prime} \alpha}\right) \cdot P\right) \times\left[t_{\gamma}, t_{j}\right] . \quad$ If $H_{2} H_{1}\left(R A_{q^{\prime} \alpha}\right)$ is contained in $Q$ then $\left(H_{3} H_{2} H_{1}\left(R A_{q^{\prime} \alpha}\right)\right) \cdot\left(B_{i} \times\left[t_{\gamma}, t_{\eta}\right]\right)=H_{3}\left(\left(R A_{q^{\prime} \alpha}\right) \cdot P \times\left[t_{j}, t_{\eta}\right]\right.$. Hence as $H_{3}(P \cdot Q)$ is between the "right" planes and the geometry of $H_{3}(Q)$ is essentially the same as $Q$, it follows that a homeomorphism $H_{1}^{\prime}$ with the properties of $H_{1}$ and a homeomorphism $H_{2}^{\prime}$ with the properties of $H_{2}$ may be defined on $\mathrm{H}_{3}(Q)$.

Further it follows that there is a homeomorphism, $\mathrm{H}_{3} \mathrm{H}_{2} \mathrm{H}_{1}$, of $E^{3}$ onto itself fixed on

$$
E^{3}-\left(\sum L A_{(p+1) \alpha}+\sum R A_{(q+1) \alpha}+\sum L A_{(p+1) \alpha}(\epsilon)+\sum R A_{(q+1) \alpha}(\epsilon)\right)
$$

taking each component of $\sum L A_{p^{\prime} \alpha}+\sum R A_{q^{\prime} \alpha}$ into a set that intersects at most four of the five planes $P_{1}, \ldots, P_{5}$. There is a homeomorphism, $H_{2}^{\prime} H_{1}^{\prime}$, fixed on $E^{3}-\left(H_{3} H_{2} H_{1}\left(\sum L A_{p^{\prime} \alpha}+\sum R A_{q^{\prime} \alpha}\right)\right)$ together with an $\epsilon / 2$ neighborhood of $H_{3}\left(\left(\sum L A_{p^{\prime} \alpha}\right) \cdot P+\left(\sum R A_{q^{\prime} \alpha}\right) \cdot P\right)$ taking each component of $H_{3} H_{2} H_{1}\left(\sum L A_{p^{\prime \prime} \alpha}+\sum R A_{q^{\prime \prime} \alpha}\right)$ into a set intersecting at most three of the five planes $P_{1}, \ldots, P_{5}$. Let $H=H_{2}^{\prime} H_{1}^{\prime} H_{3} H_{2} H_{1}$. It follows that each component of $H_{0} H\left(\sum L A_{p^{\prime \prime} \alpha}+\sum R A_{q^{\prime \prime} \alpha}\right)$ is of diameter less than $\epsilon$.

The general case follows exactly in the same way as the case where $k=5$. First we find $p^{\prime}$ and $q^{\prime}$ and define $H_{3} H_{2} H_{1}$ so that each component of $H_{3} H_{2} H_{1}\left(\sum L A_{p^{\prime} \alpha}+\sum R A_{q^{\prime} \alpha}\right)$ intersects at most $m-1$ of the $m$ planes $P_{1}, \ldots, P_{m}$. Then we find $p^{\prime \prime}$ and $q^{\prime \prime}$ and define $H_{3}^{\prime} H_{2}^{\prime} H_{1}^{\prime}$ such that each component of $H_{3}^{\prime} H_{2}^{\prime} H_{1}^{\prime} H_{3} H_{2} H_{1}\left(\sum L A_{p^{\prime \prime} \alpha}+\sum R A_{q^{\prime \prime} \alpha}\right)$ intersects at most $m-2$ of the planes $P_{1}, \ldots, P_{m}$. We then continue finding more $p$ 's and $q$ 's and defining $H_{3}$ 's and $H_{2}$ 's and $H_{1}$ 's until $H\left(\sum L A_{r \alpha}+\sum R A_{s \alpha}\right)$ intersects at most three of the $m$ planes $P_{1}, \ldots, P_{m}$, where $H$ is the composition of the $H_{3}$ 's, $H_{2}$ 's, and $H_{1}$ 's in proper order and $r$ is the last $p$ chosen and $s$ is the last $q$ chosen. We find that we have proven the lemma modulo $A(1)$, $A(2), A(3), A(4)$, and $A(5)$.

Lemma 1 is a restatement of Bing's Lemma in [3]. Following Bing we now prove the theorem. 
Proof of theorem. We find from Lemma 1 that there is a pair of positive integers $r(1)$ and $s(1)$ and a homeomorphism $T_{1}$ of $E^{3}$ onto $E^{3}$ that leaves each point of $E^{3}-\left(\sum L A_{1 \alpha}+\sum R A_{1 \alpha}+\sum L A_{1 \alpha}(1 / 2)+\sum R A_{1 \alpha}(1 / 2)\right)$ fixed and takes each component of $\sum L A_{r(1) \alpha}+\sum R A_{s(1) \alpha}$ into a set of diameter less than $1 / 2$.

There is a positive integer $\epsilon$ such that for each set $A$ of diameter less than $\epsilon, T_{1}(A)$ is of diameter less than $1 / 2^{2}$. By Lemma 1 there is a pair of positive integers $r(2)$ and $s(2)$ and a homeomorphism $T_{2}^{\prime}$ of $E^{3}$ onto $E^{3}$ that leaves each point of

$$
E^{\grave{3}}-\left(\sum L A_{r(1) \alpha}+\sum R A_{s(1) \alpha}+\sum L A_{r(1) \alpha}(\epsilon)+\sum R A_{s(1) \alpha}(\epsilon)\right)
$$

fixed and takes each component of $\sum L A_{r(2) \alpha}+\sum R A_{s(2) \alpha}$ into a set of diameter less than $\epsilon$. Let $T_{2}=T_{1} T_{2}^{\prime} . T_{2}$ is a homeomorphism of $E^{3}$ onto itself that takes each component of $\sum L A_{r(2) \alpha}+\sum R A_{s(2) \alpha}$ into a set of diameter less than $1 / 2^{2}$ and $T_{2}=T_{1}$ on

$$
E^{3}-\left(\sum L A_{r(1) \alpha}+\sum R A_{s(1) \alpha}+\sum L A_{r(1) \alpha}(1 / 2)+\sum R A_{s(1) \alpha}(1 / 2)\right) .
$$

Continuing the process we obtain a sequence of homeomorphisms $T_{1}$, $\ldots, T_{n}, \ldots$ such that $T_{n+1}=T_{n}$ on

$$
E^{3}-\left(\sum L A_{r(n) \alpha}+\sum R A_{s(n) \alpha}+\sum L A_{r(n) \alpha}\left(1 / 2^{n}\right)+\sum R A_{s(n) \alpha}\left(1 / 2^{n}\right)\right),
$$

and the diameter of each component of $T_{n+1}\left(\sum L A_{r(n+1)_{\alpha}}+\sum R A_{s(n+1) \alpha}\right)$ is less than $1 / 2^{n+1}$.

It follows that $T=\operatorname{Lim} T_{1}, T_{2}, \ldots, T_{n}, \ldots$ is a continuous function of $E^{3}$ onto $E^{3}$ such that the image of each distinct component of $L A_{0}+R A_{0}$ is a distinct point of $E^{3}$. Hence $T$ is a homeomorphism taking the decomposition space $X$ onto $E^{3}$. As $S$ is the function, defined at the end of $\$ 4$, from $\left(M+U^{1}+U^{2}\right)_{g}-y$ onto $X$, it follows that $T S$ is a homeomorphism from $\left(M+U^{1}+U^{2}\right)_{g}-y$ onto $E^{3}$ and the theorem follows with the one point compactification of $E^{3}$.

6. The details of Lemma 1 . By construction $\prod_{n=1}^{\infty}\left(\sum L A_{n \alpha}\right) \cdot P$ is a Cantor set of points in $P$. Hence for each $\epsilon>0$ there is a positive integer $n$ such that the diameter of each component of $\left(\sum L A_{n \alpha}\right) \cdot P$ is of diameter less than $\epsilon$.

We use this to find the pair of positive integers $p^{*}$ and $q^{*}$, given the pair of positive integers $p$ and $q$ and an $\epsilon>0$, as required in $A(1)$. There is a positive integer $p^{*}>p$ such that the diameter of each component of $\left(\sum L A_{p^{*} \alpha}\right) \cdot P$ is of diameter less that $\epsilon / 15$. Let $\delta$ be the minimum of $\epsilon / 15$ and the distances between pairs of components of $\left(\sum L A_{p^{*} \alpha}\right) \cdot P$. There is a positive integer $q^{*}>q+1$ such that the diameter of each component of $\left(\sum R A_{\left(q^{*}-1\right) \alpha}\right) \cdot P$ is less than $\delta$. It follows that each component of $\left(\sum L A_{p^{*} \alpha}\right) \cdot P+\left(\sum R A_{q^{*} \alpha}\right) \cdot P$ is of diameter less that $\epsilon / 5$. For let $R A_{q^{*} \alpha}$ be a component of $\sum R A_{q^{*} \alpha}$. Now $\left(R A_{q^{*} \alpha}\right) \cdot P$ is contained on the 
interior s some component of $\left.\left(\sum R A_{\left(q^{*}-1\right)_{\alpha}}\right) \cdot P\right)$. As $\delta$ was chosen so sinall that no component of $\left(\sum R A_{\left(q^{*}-1\right) \alpha}\right) \cdot P$ intersects more than one component of $\left(\sum L A_{p^{*} \alpha}\right) \cdot P, R A_{q^{* \alpha}}$ can intersect at most one component of $\sum L A_{p^{* \alpha}}$. Therefore each component of $\sum L A_{p^{* \alpha}}+\sum R A_{q^{* \alpha}}$ can contain at most one component of $\sum L A_{p^{*} \alpha}$. It follows that we may choose $p^{*}$ and $q^{*}$ with the desired properties given in $\mathrm{A}(1)$.

The positive integers $p^{\prime}$ and $q^{\prime}$ of $\mathrm{A}(2)$ are chosen in approximately the same way. Let $\delta_{1}$ be the minimum of the distance from $\left(\sum R A_{(q+3)_{\alpha}}\right) \cdot P$ to $P-\left(\sum R A_{(q+2) \alpha}\right) \cdot P$ and the distances between pairs of components of $\left(\sum R A_{(q+3) \alpha}\right) \cdot P$. There is a positive integer $r>p$ such that the diameter of each component of $\left(\sum L A_{r \alpha}\right) \cdot P$ is less than $\delta_{1}$. Note that if a component, $K$, of $\left(\sum L A_{r \alpha}\right) \cdot P$ intersects $\sum R A_{(q+3) \alpha}$ then $K$ is contained in $\operatorname{Int}\left(\left(\sum R A_{(q+2) \alpha}\right) \cdot P\right)$.

Let $\delta_{2}$ be the minimum of the distance from $\left(\sum L A_{(r+1)_{\alpha}}\right) \cdot P$ to $P-\left(\sum L A_{r \alpha}\right) \cdot P$ and the distance between pairs of components of $\left(\sum L A_{(r+1)_{\alpha}}\right) \cdot P$. There is a positive integer $s$ such that each component of $\left(\sum R A_{s \alpha}\right) \cdot P$ is of diameter less than $\delta_{2}$.

We shall now choose the $B_{i}$ 's. Let the components of $\left(\sum L A_{r \alpha}\right) \cdot P$ be called $V_{1}, V_{2}, \cdots, V_{n}$. We note that each $V_{k}$ contains two components, $U_{k, 1}$ and $U_{k, 2}$, of $\left(\sum L A_{(r+1)_{\alpha}}\right) \cdot P$. Further $\delta_{2}$ was chosen so small that no component of $\left(\sum R A_{s \alpha}\right) \cdot P$ can intersect more than one of the three sets $P-V_{k}$, $U_{k, 1}$ and $U_{k, 2}, k=1,2, \cdots, n$. Hence for each $U_{k, k}$ there is a polyhedral simple closed curve $J_{k, h}$ in $\left(\sum L A_{r \alpha}\right) \cdot P-\left(\left(\sum L A_{(r+1) \alpha}\right) \cdot P+\left(\sum R A_{s \alpha}\right) \cdot P\right)$ such that the $J_{k, h}$ 's are pair-wise disjoint, and $J_{k, h}$ separates $U_{k, h}$ from $\left(\sum L A_{(r+1)_{\alpha}}\right) \cdot P-U_{k, h}$. Let $B_{1}, \cdots, B_{2 n}$ be the polyhedral disks contained in $P$ whose boundaries are $J_{1,1}, J_{1,2}, J_{2,1}, \cdots, J_{n, 2}$. Let $B_{2 n+1}$ be a disk in $P-\sum_{i=1}^{2 n} B_{i}$ such that if $Z$ is a component of $\left(\sum R A_{s \alpha}\right) \cdot P$ that does not intersect a $B_{i}$ then $Z$ is contained in $\operatorname{Int}\left(B_{2 n+1}\right)$.

We note that each $B_{i}$ contains at most one $U_{h, k}$. As each component of $\sum L A_{(r+2) \alpha}$ intersects $P$ in the interior of some $U_{h, k}$, each $B_{i}$ intersects at most one component of $L A_{(r+2) \alpha}$ for $i \leqq 2 n$. Further each component of $\sum L A_{(r+2) \alpha}$ can intersect at most one $B_{i}$. We let $p^{\prime}=r+2$ and $q^{\prime}=s+1$, and we find that:

(i) $\left(\sum L A_{p^{\prime} \alpha}+\sum R A_{q^{\prime} \alpha}\right) \cdot P$ is contained in $\operatorname{Int}\left(\sum_{i=1}^{k} B_{i}\right)$,

(ii) at most one component of $\sum L A_{p^{\prime} \alpha}$ intersects $B_{i}, i=1,2, \cdots,(2 n+1)$,

(iii) no component of $\sum L A_{p^{\prime} \alpha}$ or of $\sum R A_{q^{\prime} \alpha}$ intersects more than one of the disks $B_{1}, \cdots, B_{(2 n+1)}$.

Suppose $B_{i}$ intersects $\sum L A_{p^{\prime} \alpha}$ and $B_{i}$ intersects $\sum R A_{q^{\prime} \alpha}$. Let $U_{k, h}$ be the component of $\left(\sum L A_{(r+1) \alpha}\right) \cdot P$ contained by $B_{i}$. It follows that $B_{i}$ is contained in $V_{k}$, a component of $\left(\sum L A_{r \alpha}\right) \cdot P$ that intersects $R A_{(q+3) \alpha}$. Therefore:

(iv) if $B_{i}$ intersects both $\sum L A_{p^{\prime} \alpha}$ and $\sum R A_{q^{\prime} \alpha}$, then $B_{i}$ is contained in $\sum \operatorname{Int}\left(\left(R A_{(q+2) \alpha}\right) \cdot P\right)$, 
(v) if $B_{i}$ intersects both $\sum L A_{p^{\prime} \alpha}$ and $\sum R A_{q^{\prime} \alpha}$, then $B_{i}$ is contained in $\sum \operatorname{Int}\left(\left(L A_{(p+1) \alpha}\right) \cdot P\right)$.

Hence the positive integers $p^{\prime}$ and $q^{\prime}$ and the disks $B_{1}, B_{2}, \cdots, B_{k}$ may be found with the properties given in $\mathrm{A}(2)$.

Before we construct $H_{0}, H_{1}$, and $H_{2}$, we note that each $L A_{n \alpha}$ is the homeomorphic image of the corresponding $C_{n \alpha}$. As $C_{n \alpha}$ is a solid right circular cylinder, there is a natural homeomorphism from $I^{2} \times[0,1]$ onto $C_{n \alpha}$. This implies that there is a natural homeomorphism from $I^{2} \times[0,1]$ onto $L A_{n \alpha}$ where $\left(I^{2} \times[0]+\left(I^{2} \times[1]\right)\right.$ is taken into $\left(L A_{n \alpha}\right) \cdot P$. We may now define a new metric, $\rho$, on $L A_{n \alpha}$ induced by the natural homeomorphism. That is, if $\psi$ is the natural homeomorphism and $x$ and $y$ are two points in $L A_{n \alpha}$, then $\rho(x, y)=d\left(\psi^{-1}(x), \psi^{-1}(y)\right)$ where $d$ is the Euclidean metric for $I^{2}$ $\times[0,1]$. Therefore, it is meaningful to speak of a piecewise linear homeomorphism of $L A_{n \alpha}$ onto itself with respect to the induced metric $\rho$. In fact the following lemma may be applied to any $L A_{n \alpha}$ using the induced metric $\rho$.

Lemma 2. Suppose $A$ is a closed subset of $I^{2} \times[0,1]$ such that $\operatorname{Bd}\left(I^{2}\right)$ $\times[0,1]$ does not intersect $A$, and $t_{1}, t_{2}, t_{3}, t_{4}, s_{1}$, and $s_{2}$ are six real numbers, $0 \leqq t_{1}<t_{2}<t_{3}<t_{4} \leqq 1, t_{1}<s_{1}<s_{2}<t_{4}$. Then there is a piece-wise linear homeomorphism of $I^{2} \times[0,1]$ onto itself fixed on $\operatorname{Bd}\left(I^{2}\right) \times[0,1]+I^{2}$ $\times\left[0, t_{1}\right]+I^{2} \times\left[t_{4}, 1\right]$ such that:

(i) the image of $(x, t)$ is $(x, s)$ where $x \in I^{2}$ and, $t$ and $s \in[0,1]$,

(ii) the image of $\left(I^{2} \times[t]\right) \cdot A$ is a subset of $I^{2} \times[s], 0 \leqq t \leqq 1$, and $0 \leqq s \leqq 1$,

(iii) the image of $\left(I^{2} \times\left[t_{2}\right]\right) \cdot A$ is contained in $I^{2} \times\left[s_{1}\right]$ and the image of $\left(I^{2} \times\left[t_{3}\right]\right) \cdot A$ is contained in $I^{2} \times\left[s_{2}\right]$.

Indication of proof. There is a disk $D$ contained on the interior of $I^{2}$ such that $A$ is contained in $D \times[0,1]$. At this point the proof follows by writing out the proper linear homeomorphism on the sets $I^{2} \times\left[0, t_{1}\right], I^{2} \times\left[t_{4}, 1\right]$, $D \times\left[t_{1}, t_{2}\right], \quad D \times\left[t_{2}, t_{3}\right], \quad D \times\left[t_{3}, t_{4}\right], \quad\left(I^{2}-\operatorname{Int}(D)\right) \times\left[t_{1}, t_{2}\right], \quad\left(I^{2}-\operatorname{Int}(D)\right)$ $\times\left[t_{2}, t_{3}\right]$, and $\left(I^{2}-\operatorname{Int}(D)\right) \times\left[t_{3}, t_{4}\right]$.

The homeomorphism promised by Lemma 2 will be called a Basic Homeomorphism. If $\phi$ is a Basic Homeomorphism of a 3 -cell, $C^{*}$, contained in $E^{3}$, then $\phi$ is a homeomorphism of $C^{*}$ onto itself and $\phi$ is fixed on $\operatorname{Bd}\left(C^{*}\right)$. Hence $\phi$ can be extended to a homeomorphism of $E^{3}$ onto itself where $\phi \mid\left(E^{3}-\operatorname{Int}\left(C^{*}\right)\right)$ is the identity.

We may now define $H_{0}$ as promised in $\mathrm{A}(3)$. By $\mathrm{A}(1)$ we may assume that the diameter of each component of $\left(\sum L A_{p_{\alpha}}+\sum R A_{q \alpha}\right) \cdot P$ is less than $\epsilon / 5$. Choose any plane $P^{\prime}$ in $L$ parallel to $P$ and at a distance of less than 1 from $P$. Choose any component of $\sum L A_{p \alpha}$, say $L A_{p \alpha}$. Let $\phi$ be a homeomorphism from $I^{2} \times[0,1]$ onto $L A_{p \alpha}$ such that $\phi\left(\left(I^{2} \times[0]\right)+\left(I^{2} \times[1]\right)\right)$ $=\left(L A_{p \alpha}\right) \cdot P$ and $\phi\left(\left(I^{2} \times\left[t_{2}\right]\right)+\left(I^{2} \times\left[t_{3}\right]\right)\right)=\left(L A_{p \alpha}\right) \cdot P^{\prime}$. As $L A_{0}$ is a Cantor set of arcs and each component of $\left(L A_{p \alpha}\right) \cdot P$ is of diameter less than $\epsilon / 5$, we may assume that the diameter of $\phi\left(I^{2} \times[t]\right)$ is less than 
$\epsilon / 5$ for $0 \leqq t \leqq 1$. Let $t_{0}=0$ and $t_{4}=1$. We have now defined the positive $\therefore$ teger: $t_{1}, t_{2}, t_{3}$, and $t_{4}$ of Lemma 2. As the diameter of $\phi\left(I^{2} \times[t]\right)$ is less than $\epsilon / 5$ for $0 \leqq t \leqq 1$ there is a pair of positive numbers $s_{1}$ and $s_{2}, 0<t_{2}$ $<s_{1}<s_{2}<t_{3}<1$, such that the diameter of $\phi\left(I^{2} \times\left[s_{1}, s_{2}\right]\right)$ is less than $\epsilon / 5$. Let the closed set of Lemma 2 be $L A_{p \alpha 1}+L A_{p \alpha 2}$. Hence we apply Lemma 2 and the construction of $H_{0}$ is obvious when we note that the Basic Homeomorphism is uniformly continuous.

We shall now define $H_{1}$ as promised in $A(4)$. Suppose $L A_{(p+1) \alpha}$ is any component of $\sum L A_{(p+1) \alpha}$ and $L A_{p^{\prime} \alpha}$ is a component of $\left(\sum L A_{p^{\prime} \alpha}\right) \cdot\left(L A_{(p+1) \alpha}\right)$. Let $P_{1}$ and $P_{2}$ be defined as in $\mathrm{A}(3)$. Let $R_{1}$ and $R_{2}$ be the closure of the two components of $L A_{(p+1) \alpha}-P_{1}$ that intersects both $P_{1}$ and $P$. As $R_{i}$, $i=1,2$, is a right cylinder, there is a homeomorphism $\phi_{1}$ of $I^{2} \times[0,1]$ such that:

(i) $\phi_{1}\left(I^{2} \times\left[0, t_{1}\right]=R_{1}\right.$,

(ii) $\phi_{1}\left(I^{2} \times\left[t_{3}, 1\right]\right)=R_{2}$,

(iii) $\phi_{1}\left(I^{2} \times[t]\right), 0<t<t_{1}, t_{3}<t<1$, is a disk contained in a plane parallel to $P$ which irreducibly separates $L A_{p \alpha}$.

It follows that $P_{2} \cdot\left(L A_{(p+1) \alpha}\right)=\phi_{1}\left(I^{2} \times\left[t_{5}\right]\right)+\phi_{1}\left(I^{2} \times\left[t_{4}\right]\right)$ for some $t_{5}$ and $t_{4}, 0<t_{5}<t_{1}<t_{3}<t_{4}<1$. We may assume without loss of generality that $L A_{p^{\prime} \alpha}=L A_{(p+1) \alpha, 1,1, \cdots, 1,1}$. Let $R_{1} \cdot\left(L A_{(p+1) \alpha 1}\right) \neq \emptyset$. There is a pair of positive numbers $s_{1}$ and $s_{2}$ such that $t_{3}<s_{1}<s_{2}<t_{4}$. By construction $\left(\phi_{1}\left(I^{2} \times\left[t_{1}\right]\right)\right) \cdot\left(L A_{(p+1) \alpha 2}\right) \neq \emptyset$. Hence there is a positive number $t_{2}, t_{1}$ $<t_{2}<t_{3}$ such that $\left(\phi_{1}\left(I^{2} \times\left[t_{2}\right]\right)\right) \cdot\left(L A_{(p+1) \alpha 2}\right)=\emptyset$. We have chosen the six positive numbers $t_{1}, t_{2}, t_{3}, t_{4}, s_{1}$, and $s_{2}$. Hence there is a Basic Homeomorphism $\psi_{1}$ as defined in Lemma 2 such that:

(i) $\psi_{1}$ is fixed on the closure of the two components of $L A_{(p+1) \alpha 1}-P_{1}$ that intersect both $P_{1}$ and $P$,

(ii) $\psi_{1}$ is fixed on that part of $L A_{(p+1) \alpha}$ between $P_{2}$ and $P$,

(iii) $\psi_{1}$ is fixed on $E^{3}-L A_{(p+1) \alpha}$,

(iv) $\psi_{1}\left(L A_{(p+1)_{2} 2}\right)$ does not intersect $P_{1}$. If $L A_{p^{\prime} \alpha}=L A_{(p+1) \alpha 1}$ let $\psi_{1}=H_{1}$.

Suppose $L A_{p^{\prime} \alpha} \neq L A_{(p+1) \alpha 1}$. By (i) in the above paragraph we note that the closure of the two components of $\psi_{1}\left(L A_{(p+1) \alpha 1}\right)-P_{1}$ that intersect both $P$ and $P_{1}$ are right cylinders. We also note that the only condition for the definition of $\psi_{1}$ was that $R_{1}$ and $R_{2}$ be right cylinders. It follows that a homeomorphism $\psi_{2}$ with properties given for $\psi_{1}$ in the above paragraph may be defined by substituting $\psi_{1}\left(L A_{(p+1) \alpha 1}\right)$ for $L A_{(p+1) \alpha}$. If $L A_{p^{\prime} \alpha}$ $=L A_{(p+1) \alpha 1,1}$ let $H_{1}=\psi_{2} \psi_{1}$. If $L A_{p^{\prime} \alpha} \neq L A_{(p+1) \alpha 1,1}$, then there is a homeomorphism $\psi_{3}$. It follows that in a finite number of steps the homeomorphism $H_{1}$ will be defined with the properties of $\mathrm{A}(4)$. Further as the homeomorphism $H_{3} H_{1}$ takes the closure of the two components of $L A_{n \alpha}-P_{1}$ that intersects both $P$ and $P_{1}$ into right cylinders the homeomorphism $H_{1}^{\prime}$ may be defined. 
The homeomorphism $H_{2}$ given in $\mathrm{A}(5)$ is defined in exactly the same way as $\psi_{1}$ where $L A_{(p+1) \alpha}$ is replaced by $R A_{(q+1) \alpha}, P_{1}$ is replaced by $P_{n}$, and $P_{2}$ is replaced by $P_{(n-1)}$.

We have supplied the details of Lemma 1 and have completed the proof of the theorem.

\section{BiBLIOGRAPHY}

1. J. W. Alexander, An example of a simply connected surface bounding a region which is not simply connected, Proc. Nat. Acad. Sci. U.S.A. 10 (1924), 8-10.

2. B. J. Ball, The sum of two solid horned spheres, Ann. of Math. (2) 69 (1959), 253-257.

3. R. H. Bing, A homeomorphism between the 3-sphere and the sum of two solid horned spheres, Ann. of Math. (2) 56 (1952), 354-362.

4. W. A. Blankinship and R. H. Fox, Remarks on certain pathological open subsets of 3space and their fundamental group, Proc. Amer. Math. Soc. 1 (1950), 618-624.

Louisiana State University,

Baton Rouge, Louisiana 